\title{
¿Son los ácidos grasos de la carne y la leche bovina nocivos para la salud de las personas?
}

\author{
Are fatty acids from beef and \\ milk from cattle harmful \\ for human health?
}

\begin{abstract}
Nutritional recommendations of the past 35-40 years have promoted the reduction of fat intake, calories and particularly saturated fatty acids (SFA), emphasizing lower consumption of red meat and high fat dairy products, because of its association with cardiovascular diseases (CVD), being in turn replaced by sugars and refined carbohydrates. There is consensus that the SFA concentration in blood plasma, particularly palmitic acid, are associated with an increased risk of CVD and heart attacks. However, there is no evidence of causality between CVD and consumption of SFA, raising reasonable doubts about the relationship between consumption of SFA with those present in blood plasma, as well as with the CVD risk. This review presents scientific evidence that demonstrates that the inclusion of fats in the diet, specifically animal fats from ruminants, provide important health benefits for people, especially when accompanied by a reduction in the consumption of carbohydrates. It is also proposed that ruminant products and their fatty acids contribute with beneficial saturated fats for people's health, especially those from pastoral production systems such as vaccenic, rumenic and linolenic acids.

Key words: omega-3, cholesterol, congujated linoleic acid.
\end{abstract}

Rodrigo Arias (1) Juan Pablo Keim (1) Alejandro Velásquez (2) Einar Vargas-Bello-Pérez (3)

(1) Instituto de Producción Animal, Facultad de Ciencias Agrarias, Universidad Austral de Chile. Valdivia, Chile. (2) Escuela de Agronomía y Núcleo de Investigación en Producción Agroalimentaria, Universidad Católica de Temuco Departamento de Ciencias Animales, Temuco, Chile. (3) Facultad de Agronomía e Ingeniería Forestal, Pontificia Universidad Católica de Chile, Santiago, Chile.

Dirigir la correspondencia a: Profesor Rodrigo Arias rodrigo.arias@uach.cl

Este trabajo fue recibido el 25 de Noviembre de 2015 aceptado con modificaciones el 27 de Agosto de 2016 y aceptado para ser publicado el 12 de Octubre de 2016.

\section{INTRODUCCIÓN}

Las recomendaciones nutricionales de los últimos 35 a 40 años han promovido una reducción del consumo de carnes rojas y productos lácteos por su contenido de ácidos grasos saturados (AGS), bajo la premisa fundamental de reducir el riesgo de enfermedades cardiovasculares (ECV). Estas recomendaciones se basan en la hipótesis de los lípidos, desarrollada por Ancel Keys luego de publicar su estudio de los 7 países (1), que cambió de manera sustantiva la dieta de la humanidad (2). Desde entonces se han realizado numerosos estudios evaluando el efecto de distintos tipos de ácidos grasos sobre la salud de las personas. La reducción de la ingesta de ácidos grasos saturados y de colesterol dietario, y en parte sustituyendo estos por ácidos grasos de tipo mono/poliinsaturados (AGM y AGPI, respectivamente), han resultado en una reducción tanto del colesterol sérico como del riesgo de desarrollar ECV (3). No obstante, durante los últimos años el rol de los ácidos grasos saturados e insaturados se ha modificado progresivamente.
Mcguire y Mcguire (4) destacaron el rol benéfico de ácidos grasos de origen ruminal en la salud de las personas, mientras que Ascherio y Willett (5) plantearon efectos negativos por el consumo de ácidos grasos de tipo trans. Todas las evidencias recientes indican que ciertos ácidos grasos ingeridos en la dieta juegan un papel importante en la causa y la prevención de las ECV, pero la grasa total como porcentaje de la energía consumida no es importante. $(3,6,7)$. Esto último es respaldado por el reporte de la FAO (8), planteando que la distribución energética de los macronutrientes no es relevante como si lo es el lograr un adecuado equilibrio energético.

En el mismo periodo (últimos 35 a 40 años) la industria alimentaria ha sustituido las grasas por aceites vegetales y el azúcar con el objeto de mejorar el sabor y palatabilidad de los productos. Entre los productos utilizados como endulzantes, uno de los más utilizados por su bajo costo es el jarabe de maíz de alta fructosa (High Fructose Corn Syrup), cuya molécula es bastante similar a la del azúcar. Los trabajos del grupo de 
investigación del Dr. Johnson de la Universidad de Colorado, han demostrado que la fructosa gatilla en el organismo el almacenamiento de grasa y la resistencia a la insulina. En la actualidad se observa azúcar agregada en más del 75\% de los productos alimenticios comercializados en Estados Unidos (9), y probablemente del resto del mundo dada la existencia de las grandes compañías transnacionales Sin embargo, a la fecha las ECV siguen siendo la principal causa de muerte a nivel global (10) sumando además un importante aumento de la población mundial con sobrepeso y obesidad, desde valores del $29 \%$ en 1980 al 37\% en el 2013 (11). Esto último se explicaría por el mayor consumo de alimentos procesados (12), los cuales son ricos en azúcares. En el caso de Chile, al año 2009, un 39,3\% de la población adulta presentaba sobrepeso y 25,1\% obesidad (13) y según Azar et al. (14) se concentran mayoritariamente en personas de escasos recursos y bajo nivel de educación. La obesidad se ha convertido en el mayor desafío de la salud mundial por su importante aumento en los últimos 35 años, y porque en el mismo periodo no se registran éxitos en su control y reducción (11).

Durante los últimos 5 años y de forma cada vez más frecuente, se ha cuestionado con mayor frecuencia la validez y éxito de las actuales recomendaciones nutricionales (15), las que han resultado básicamente en la sustitución de grasas por carbohidratos para mantener el equilibrio energético. Esto último, según lo planteado por la FAO (8) y por Willett (7), no genera beneficio en la salud de las personas, existiendo evidencia convincente de que la sustitución de AGMI por carbohidratos aumenta el nivel de colesterol HDL-C y que por el contrario la sustitución de carbohidratos por AGMI mejora la sensibilidad a la insulina. La FAO (8) plantea que la sustitución de AGS por carbohidratos muy refinados no tiene beneficios sobre las ECV, pudiendo incluso incrementar y favorecer el desarrollo del síndrome metabólico.

Finalmente, Lamarche y Couture (16) plantean la necesidad de revisar las recomendaciones dietarias respecto al consumo de grasas en general y de grasas saturadas en particular. El objetivo de esta revisión fue investigar la relación existente entre el consumo de ácidos grasos saturados particularmente aquellos de origen animal (rumiantes) y la salud de las personas, en base a la literatura científica reciente.

\section{Alimentación y mortalidad en Chile}

La dieta de los chilenos corresponde mayoritariamente a carbohidratos y azúcares (73,6\% del total), mientras que las grasas y proteína representan el 13,3\% y 10,4\%, respectivamente. Las grasas saturadas constituyen $31,7 \%$ del total de grasas consumidas o bien 4,2\% sobre el total de la dieta (17). Sin embargo, no existen datos que permitan determinar el origen de las mismas. Destaca el alto consumo anual de pan (86,5 kg/persona) en relación con otros países de la Región y a nivel mundial (18). Cabe enfatizar la reducción, aunque leve, en el consumo de azúcar durante el decenio 2003-2013 (1,7\% anual), lo que está en línea con la recomendación de la OMS que indica que el azúcar libre sea $<10 \%$ del total de la energía consumida (19).

El consumo aparente de carnes en Chile en el decenio 2003-2013 aumentó tanto en porcinos como aves (3,4\% y $3,1 \%)$, mientras que la carne bovina presentó un crecimiento de tan sólo 0,5\% anual (20). Las dos primeras son consideradas por la población como las más sanas por su menor tenor de grasas saturadas. Además, son más accesibles por su menor costo y el menor tiempo que demanda su producción. Por otra parte, el consumo de productos lácteos aumentó casi $11 \%$ durante los últimos cinco años, siendo los yogures y la leche cultivada el segmento de mayor crecimiento para 2006-2011, con 35,4\% (21). El alto consumo de yogurt (14,5 L per cápita en 2012) posiciona a Chile como el mayor de Sudamérica y sobre Estados Unidos y Canadá. Los yogures son considerados mayoritariamente como saludables por la población, aun cuando pueden presentar altos contenidos de azúcar y ser bajos en grasa total (productos light). En el mismo contexto, la producción de leche descremada y semi-descremada (tanto fluida esterilizada como en polvo) ha aumentado entre 33 y $1000 \%$ entre 2010 y 2014, lo que ocurre a mayor razón que la recepción de leche $(13,4 \%)$, elaboración de leche esterilizada entera $(9,4 \%)$ y leche en polvo con $28 \%$ materia grasa $(-41,4 \%)(20,22)$. Esta situación sugiere una tendencia hacia un mayor consumo de lácteos con menor contenido de grasa por parte de los consumidores chilenos, siguiendo las directrices nutricionales.

Durante el periodo 2000-2011, la primera causa de muerte en Chile correspondió a enfermedades del sistema circulatorio (23) seguida por tumores malignos, representando aumentos de $4,7 \%$ y $15,7 \%$ (figura 1). Destaca la mortalidad por enfermedad isquémica del corazón y las cerebro-vasculares (47,9 y 48,8 por cada 100.000 habitantes). En tanto la Organización Panamericana de la Salud señala que sobre $63 \%$ de los adultos $>17$ años presentan un colesterol total elevado (> $200 \mathrm{mg} / \mathrm{dl}$ ) y HDL-C disminuido (<40 mg/dl). La reducción del consumo de colesterol ha sido uno de los principales objetivos dietarios dada su asociación con las ECV y se encuentra muy arraigado en la población, al igual que el concepto de colesterol bueno y malo (HDL-C y LDL-C). Si bien el colesterol es el principal componente de la placa aterosclerótica (24), también cumple diversas funciones en el organismo: incluyendo el desarrollo del cerebro y procesos de curación (25), formando parte de las membranas celulares y como base para la síntesis de Vitamina D y hormonas esteroidales. Sin embargo, a diferencia de los triglicéridos, este no es utilizado como fuente energía en el cuerpo. La mayoría del colesterol en el plasma sanguíneo se sintetiza de forma endógena, siendo la ingesta menos importante de lo pensado originalmente (26). Por ello, la estrategia de reducir el consumo de colesterol como alternativa para minimizar el riesgo de ECV sería efectiva sólo en una proporción menor de la población (27). En este mismo sentido, el Comité Asesor de Directrices Nutricionales de Estados Unidos ha señalado (2015) que el sobreconsumo de colesterol no es tema de preocupación (28), basado en la falta de evidencia que muestre alguna relación entre el consumo de colesterol dietario con colesterol sérico. Por otra parte, Krauss (29) sugirió la existencia de dos patrones para el LDL-C (A y B), presentando estos un mayor poder predictivo en el riesgo de ECV que la sola medición de LDL-C (30). En consecuencia, las partículas pequeñas y densas (LDL-C patrón B) presentan un mayor riesgo cardiovascular que las del patrón $A$, menos densas y más grandes (31).

\section{Salud, consumo de productos animales y ácidos grasos}

El efecto de los ácidos grasos en la salud de las personas ha sido sujeto de numerosas investigaciones durante las últimas décadas. Al respecto, recientemente la FAO (8) publicó un reporte de un panel de expertos del consumo de grasas y ácidos grasos, reconociendo que los ácidos grasos individuales dentro de cada grupo (AGS, AGMI y AGPI) pueden tener propiedades biológicas y efectos específicos sobre la salud. A la fecha existe consenso de que la concentración de AGS 
en el plasma sanguíneo, particularmente del ácido palmítico (C16:0) se asocia con un mayor riesgo de ECV y ataques al corazón (6). Sin embargo, no existe evidencia que permita concluir que el consumo de AGS esté asociado a un mayor riesgo de ECV (32). Lo anterior plantea dudas razonables sobre la relación entre el consumo de AGS, los presentes en el plasma sanguíneo y el riesgo de ECV. Por el contrario, dietas con mayores contenidos de AGS han demostrado disminuir su concentración en el plasma sanguíneo, particularmente de C16:0, y aumentando al mismo tiempo la concentración de AGPI, especialmente C18:2 (33). Asimismo, King et al. (34), reportaron incrementos de 11 a $13 \%$ en C16:0, reducciones de 25 a 35\% en AGMI y aumentos de 2 a $8 \%$ en AGPI (disminución de 11 a 13\% de C18:2 $\omega-6$ ) en dietas bajas vs. dietas moderadas en grasas saturadas. Por su parte, Forsythe et al. (35) reportaron que dietas muy bajas en carbohidratos (12\% total) y altas en grasas (59\%), provocaron importantes alteraciones en la composición de ácidos grasos sanguíneos, reduciendo el total de éstas y la concentración de C16:1 $\omega-7$ en mayor proporción respecto una dieta rica en carbohidratos $(56 \%)$ y baja en grasas totales (24\%). Lo anterior implica una menor biosíntesis de novo de lípidos en la dieta baja en carbohidratos y alta en grasas saturadas. Otros estudios reportan un aumento tanto el LDL-C y HDL-C en dietas con mayor contenido de grasas saturadas, mientras que el consumo de grasas insaturadas disminuye el LDL-C y los triglicéridos, aumentando el HDL-C (36). El consumo de AGS, AGMI y AGPI elevan el HDL-C cuando reemplazan a los carbohidratos en la dieta, y su efecto es ligeramente superior en dietas con mayor contenido de AGS (37). Esto implica que los AGS en el plasma sanguíneo no provienen de la grasa consumida, sino más bien del exceso de carbohidratos y azúcares que son removidos del plasma por la insulina y convertidos en grasa. Esto explicaría las mayores concentraciones de C16:0 y C16:1 $\omega-7$, ambos provenientes de la síntesis de novo de lípidos en las personas.

En tanto los ácidos grasos trans son considerados nocivos para la salud, pues elevan LDL-C, disminuyen HDL-C e incrementan la inflamación (25), con la excepción de aquellos encontrados en productos originados en rumiantes, como la leche, quesos, mantequilla y carne (C18:1 t-11 y C18:2 c-9, t-11): Estos ácidos grasos elevan los valores de HDL-C en modelos animales (38), presentan efectos neutrales en personas (39) y poseen propiedades anticarcinogénicas y antiaterogénicas (40). Por su parte, Chardigny et al. (41), comparando sujetos consumiendo ácidos grasos trans naturales (rumiantes) vs. trans industrializados, concluyeron que los primeros favorecieron las concentraciones de subfracciones de LDL-C patrón $A$, es decir, menos riesgoso; mientras los de tipo trans industrial aumentaron las concentraciones de LDL-C patrón B.

Los alimentos provenientes de sistemas de producción animal presentan una alta variación respecto al contenido graso, perfil de ácidos grasos y colesterol. La tabla 1 presenta una comparación del perfil de ácidos grasos, calorías y colesterol de distintas carnes y productos lácteos tanto de origen nacionales e internacional, ya sea cocidas o frescas (cruda).

Productos lácteos. La leche de rumiantes tiene alrededor de 400 ácidos grasos, siendo el contenido de grasa y la composición en la leche dependiente de diversos factores como la dieta y nutrición recibida por el animal, estado fisiológico, genética, nivel productivo, entre otros $(42,43)$. Actualmente hay consenso en que no todos los ácidos grasos tienen el mismo potencial colesterolémico. Los ácidos grasos de cadena corta (C4:0, C6:0, C8:0 y C10:0), ácido esteárico (C18:0) y AGMI no afectarían las concentraciones de colesterol sérico. Por otro

\section{FIGURA 1}

Tasas de mortalidad por cada 100.000 habitantes en Chile, periodo 2000-2011. Elaborado a partir de los datos del Departamento de Estadísticas e Información de Salud (www.deis.cl).

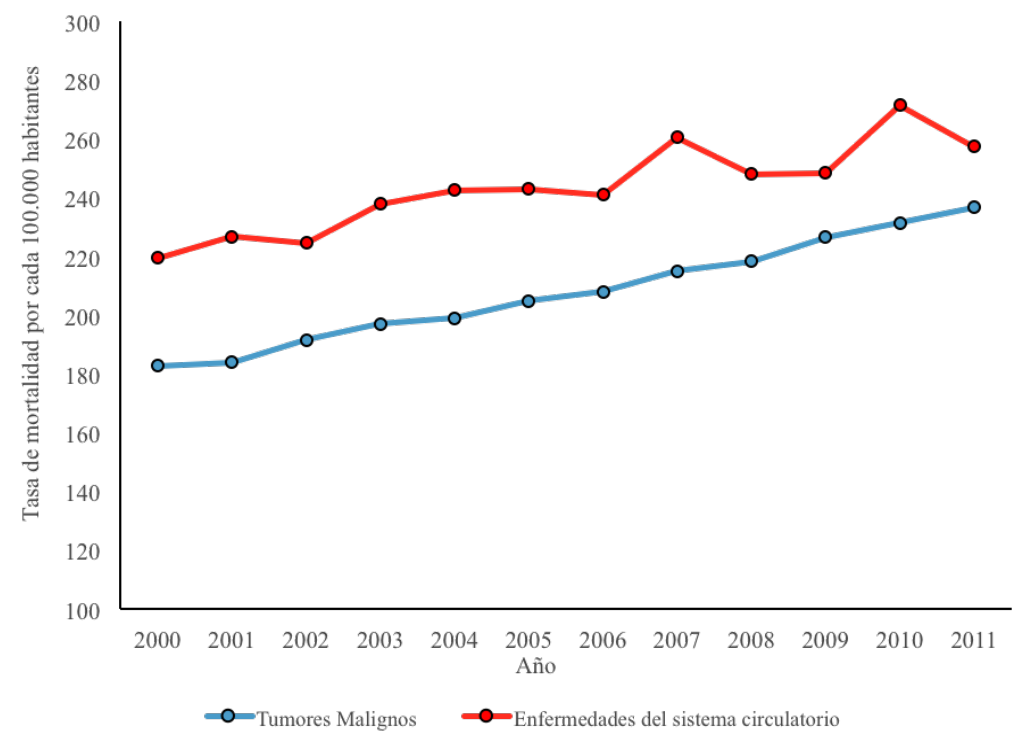


lado, algunos ácidos grasos como el butirato, ácido linoléico conjugado (ALC) tienen el potencial de prevenir cáncer y aterogenia $(44,45)$. Sin embargo, otros ácidos grasos como el laúrico, palmítico y mirístico tienen efectos negativos en la salud (46). Los isómeros que constituyen los ALC se producen naturalmente en los rumiantes como productos intermediarios de la biohidrogenación del ácido linoleico y que resultan en la generación de una mezcla de AGMI y AGS. Además, los ALC pueden sintetizarse a partir de la desaturación del ácido vaccénico (C18:1 trans-11) en los rumiantes y personas. En consecuencia, la principal fuente natural de ALC para las personas son los productos cárnicos, grasos y lácteos derivados de los rumiantes (tabla 2), aunque también es posible obtener ALC en forma sintética a través de la hidrogenación industrial del aceite de soya, maíz o cártamo (47). Entre las propiedades de los ALC en leche y queso bovino destacan sus efectos antiaterogénico y antitrombogénico (48), al igual que el de origen ovino (49).

Los productos lácteos constituyen una parte importante de la dieta humana, con cerca del $75 \%$ proveniente de rumiantes (50) y en su mayoría de la leche bovina. Los ácidos grasos trans del rumen son incorporados en la grasa de la leche (51), representando una mezcla de diferentes isómeros. En consecuencia, el queso, mantequilla, leche y carne de vacunos contienen entre 2 y $8 \%$ de ácidos grasos trans de su peso total (52). No obstante el reconocido efecto benéfico de estos ácidos grasos trans de rumiantes el Comité de expertos de la OMS propuso disminuir su ingesta a menos de $1 \%$ de la energía total ingerida para prevenir ECV (53), recomendación que ha sido seguida en Chile. En tanto, otros

TABLA 1

Contenidos de grasa total, ácidos grasos saturados (AGS), monoinsaturados (AGMI) y poliinsaturados (AGPI) y colesterol de diversos productos cocidos y frescos (g/100 g ácidos grasos totales).

Elaborado a partir de los datos USDAt, Larraín y Vargas-Bello-Pérez, y Morales et al. (2015)

\begin{tabular}{|c|c|c|c|c|c|c|}
\hline Alimento & $\begin{array}{l}\text { Calorías } \\
\text { (100 g) }\end{array}$ & $\begin{array}{l}\text { Grasa } \\
\text { Total }\end{array}$ & AGS & $\begin{array}{l}\text { AGMI } \\
\text { g/100g }\end{array}$ & AGPI & $\begin{array}{c}\text { Colesterol } \\
(\mathrm{mg})\end{array}$ \\
\hline Carne molida (10\% grasa, cocida) & 214,0 & 11,10 & 4,38 & 4,67 & 0,40 & 88,0 \\
\hline Pollo ganso (1/8" grasa, Select, cocida) & 159,0 & 7,57 & 2,98 & 3,27 & 0,29 & 66,0 \\
\hline Lomo liso (1/8" grasa, Select, cocida) & 200,0 & 8,39 & 3,20 & 3,35 & 0,32 & 82,0 \\
\hline Lomo vetado (1/8" grasa, Select, cocida) & 192,0 & 8,48 & 3,53 & 4,02 & 0,54 & 77,0 \\
\hline Entrecot cocido (1/8" grasa, Select cocida) & 206,0 & 9,35 & 3,65 & 3,79 & 0,48 & 83,0 \\
\hline Pechuga de pollo (con piel) al horno & 197,0 & 7,78 & 2,19 & 3,03 & 1,66 & 84,0 \\
\hline Pechuga de pollo (sin piel) al horno & 165,0 & 3,57 & 1,01 & 1,24 & 0,77 & 85,0 \\
\hline Filete cerdo (cocido) & 147,0 & 3,96 & 1,36 & 1,52 & 0,57 & 73,0 \\
\hline Salmon cocido (Chinook) & 231,0 & 13,38 & 3,21 & 5,74 & 2,66 & 85,0 \\
\hline Atún al agua (White) & 128,0 & 2,97 & 0,79 & 0,78 & 1,11 & 42,0 \\
\hline Atún en aceite (White) & 186,0 & 8,08 & 1,28 & 3,26 & 2,97 & 21,0 \\
\hline Leche entera $(3,25 \% M G)$ & 61,0 & 3,27 & 1,87 & 0,81 & 0,20 & 10,0 \\
\hline Leche $2 \%$ MG & 50,0 & 1,98 & 1,26 & 0,56 & 0,07 & 8,0 \\
\hline Leche 1\% MG & 42,0 & 0,97 & 0,63 & 0,28 & 0,04 & 5,0 \\
\hline Leche sin grasa & 34,0 & 0,08 & 0,05 & 0,02 & 0,003 & 2,0 \\
\hline Queso cheddar & 533,0 & 43,97 & 24,90 & 12,21 & 1,88 & 131,0 \\
\hline Queso blanco & 310,0 & 24,31 & 13,66 & 6,46 & 1,15 & 70,0 \\
\hline Queso parmesano rayado & 420,0 & 27,84 & 15,37 & 7,13 & 1,39 & 86,0 \\
\hline Queso Brie & 334,0 & 27,68 & 17,41 & 8,01 & 0,83 & 100,0 \\
\hline Queso Camembert & 300,0 & 24,26 & 15,26 & 7,02 & 0,72 & 72,0 \\
\hline Queso Crema & 350,0 & 34,44 & 2,93 & 1,29 & 0,22 & 15,0 \\
\hline Leche cruda (productor 3,9\% MG) & 68,1 & 3,84 & 2,61 & 1,12 & 0,14 & nd \\
\hline Yogurt (de leche entera) & 61,0 & 3,25 & 2,10 & 0,89 & 0,09 & 13,0 \\
\hline Yogurt (bajo en grasa) & 63,0 & 1,55 & 1,00 & 0,43 & 0,044 & 6,0 \\
\hline Mantequilla con sal & 717,0 & 81,11 & 51,37 & 21,02 & 3,04 & 215,0 \\
\hline Lomo liso chileno (fresco) & 108,2 & $2,18 \#$ & 0,968 & 0,774 & 0,256 & 47,1 \\
\hline Lomo vetado chileno (fresco) & 128,8 & $5,13 \#$ & 2,36 & 1,686 & 0,656 & 50,6 \\
\hline Sobrecostilla chilena (fresco) & 116,5 & $3,74 \#$ & 1,455 & 1,239 & 0,736 & 51,8 \\
\hline Cerdo, chuleta de centro chilena (fresco) & 118,8 & 3,86\# & 0,846 & 1,547 & 1,117 & 51,3 \\
\hline Salmon filete chileno (fresco) & 193,4 & $12,66 \#$ & 4,099 & 3,59 & 2,28 & 49,2 \\
\hline Truto entero sin piel chileno (fresco) & 100,6 & 4,09\# & 1,053 & 1,43 & 1,371 & 67,4 \\
\hline
\end{tabular}


países como Canadá permiten hasta 5\% de éstos ácidos grasos trans en los alimentos. Los sistemas de producción de leche en Chile han mostrado tener algún efecto sobre el perfil de ácidos grasos de la leche. Morales et al. (54) reportaron que los sistemas pastoriles y mixtos mejoran la concentración de ácidos grasos $\omega$-3 y del ácido ruménico al final del invierno y durante la primavera. No obstante, no se reportó un efecto del sistema productivo por sí solo ni en el contenido de AGS ni de insaturados (AGMI + AGPI), sino más bien un efecto de interacción del tiempo y del sistema productivo.

Productos cárnicos. Otros ácidos grasos producidos en el rumen como el vaccénico y ruménico, han recibido mucha atención dado que tienen efectos positivos en la salud humana, como lo es la reducción de resistencia a la insulina, la cual se asocia a ECV. La carne bovina es una fuente importante de éstos ácidos grasos (ambos trans) y de los ALC (55). Diversos investigadores han demostrado que el ácido vaccénico reduce la citoquinas pro-inflamatorias $(56,57)$ y la agregación plaquetaria en humanos y los triglicéridos en plasma en modelos animales $(58,59)$. En tanto el ácido ruménico reduce el riesgo de ECV y cáncer, incrementa la masa ósea y modula la respuesta inmune e inflamatoria (60). Asimismo, las concentraciones de estos ácidos grasos benéficos aumentan cuando los animales son alimentados en base a forrajes (55), como es el caso de la gran mayoría de la carne producida en Chile (61). La figura 2 muestra el perfil de ácidos grasos y colesterol de carnes y

TABLA 2

Principales fuentes de ácido linoleico conjugado (ALC).

Alimento

Leche entera

Leche semidescremada ( $2 \%$ de grasa)

Leche condensada

Mantequilla

Yogurt

Yogurt bajo en grasa

Queso tipo Cheddar

Carne de bovino

Carne de ovino

Carne de ternera
Cantidad de ALC (g/100 g de grasa)
0,55
0,41
0,70
0,47
0,48
0.44
0,41
0.43
0,58
0,27

Modificado de Roche (71).

\section{FIGURA 2}

Contenido ácidos grasos saturados (AGS; g/100 g), monoinsaturados (AGMI; g/100 g) y poliinsaturados (AGPI; g/100 g), ácido ruménico (AR; $\mathrm{mg} / 100 \mathrm{~g}$ ) y colesterol (mg/100 g) en: a) Leche cruda y b) Músculo Longissimus thoracis de novillos, ambos en distintos sistemas de producción. Elaborado a partir de Morales et al. (2012; 2015).
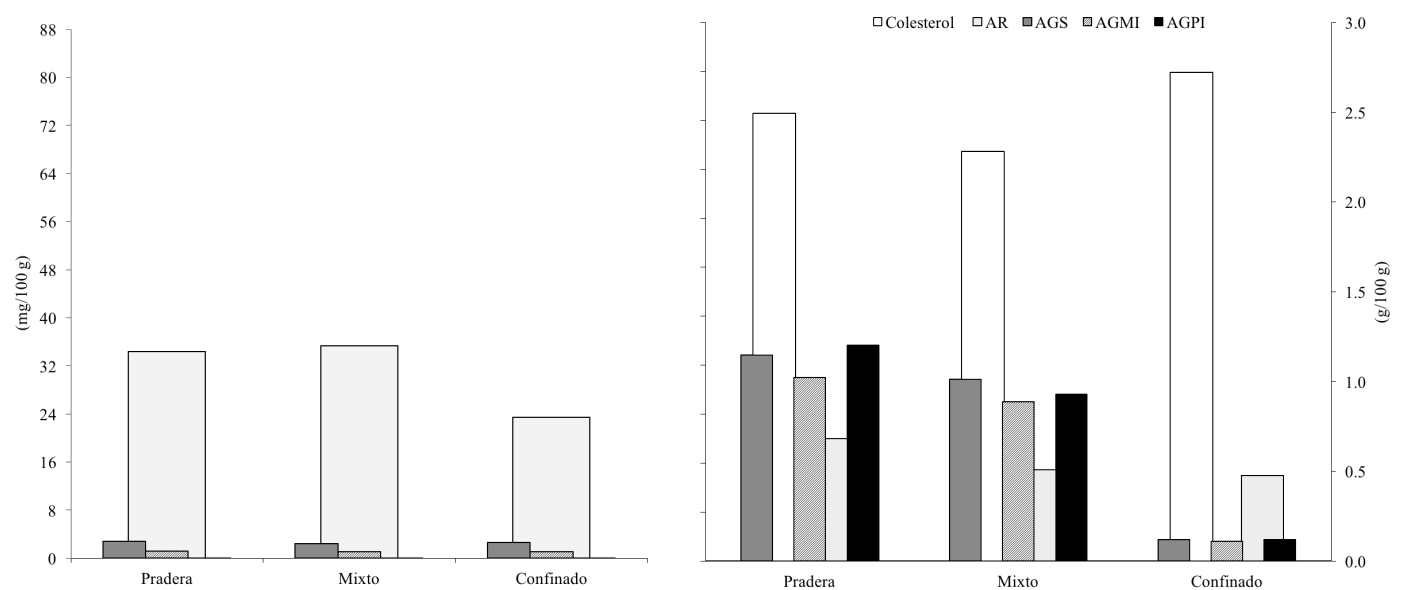
leche fresca producidos en Chile $(54,61)$. No se observan diferencias importantes en términos de la contribución de estos en ninguno de los sistemas de producción tradicionales del país. Sin embargo, se observa que tanto la leche como la carne que provienen de sistemas pastoriles presentan un mayor contenido de ácido ruménico, lo que estaría relacionado con alimentos más saludables. Asimismo, Larraín y Vargas-BelloPérez (62) plantean que las carnes nacionales son extra magras (salvo el lomo vetado) y muy saludables. No observándose diferencias con los valores de otras carnes como la chuleta de cerdo y trutro de pollo.

En el caso de la carne rojas y en particular de las carnes magras, Roussell et al. (63), las consideran saludables pues reducen los factores de riesgo de ECV, siendo comparables a los provocados por una dieta DASH (Diet Approaches to Stop Hypertension). Los autores incluyeron 4 dietas isocalóricas: HAD (Health American Diet); BOLD (Beef Optimum Lean Diet); DASH; y BOLD+ (similar a BOLD pero con menos carbohidratos y más proteína), logrando en todas ellas, salvo HAD, reducir el colesterol total, LDL-C y HDL-C. Cabe señalar que dicho estudio presentó valores elevados de carbohidratos totales en las dietas alcanzando 50\%, 54\%, 55\% y 45\%, respectivamente. Por su parte, Haring et al. (64) plantean que el consumo de proteína animal no incrementa el riesgo de ECV. En tanto, Scott et al. (65) reportaron que la inclusión de carne de pollo o vacuno en la dieta reducen el colesterol total y LDL-C de manera similar. En otro estudio, Gilmore et al. (66), evaluaron el consumo de hamburguesas de carne vacuna durante 5 semanas con una grasa total de $24 \%$ pero con una relación AGMI:AGPI de 0,71 vs. 1,10 (proveniente de animales finalizados en pradera o feedlot, respectivamente). Los autores reportaron que ambos tratamientos disminuyeron la insulina en plasma y los diámetros de partículas de HDL-C2 y HDL-C3, y al mismo tiempo aumentaron la concentración en plasma de ácido esteárico (C18:0) y araquidónico (C20:4 $\omega-6)$ respecto de los valores basales. Asimismo, indicaron que sólo la carne proveniente de feedlot aumentó el HDL-C y reportaron una correlación negativa de HDL-C y C18:0 con las concentraciones de triglicéridos e insulina en plasma. En tanto la correlación fue positiva entre estas dos últimas.

\section{Dietas en base a ácidos grasos} saturados vs. carbohidratos

Uno de los resultados plausibles de las recomendaciones dietarias ha sido la reducción del consumo de carnes rojas y productos lácteos sin descremar, pero al mismo tiempo reemplazadas por más carbohidratos refinados y azúcar. Esto a su vez causa una elevada concentración de triglicéridos en plasma (67). La evaluación de dietas de tipo cetogénicas (ricas en grasas totales naturales y bajas en carbohidratos), demuestran una mejora en todos los indicadores asociados al síndrome metabólico (68). Este síndrome se caracteriza por un aumento del peso, grasa total, triglicéridos, glucosa, insulina y presión sanguínea, mientras que se reduce el valor de HDLC. En tanto Volek et al. (69) han demostrado que una dieta restringida de carbohidratos (12\% vs. 56\%) mejora de manera importante la concentración de glucosa e insulina, sensibilidad a la insulina, pérdida de peso, adiposidad, triglicéridos, HDL-C y relación colesterol total/HDL-C. En cambio, una dieta alta en carbohidratos produce intolerancia a los carbohidratos (insulina) aumentando los ácidos grasos C16:0 y C16:1 $\omega$-7 en plasma. Por su parte, Faghihnia et al. (70) evaluaron el efecto de una dieta baja en carbohidratos (31\%), alta en proteína de origen bovino (31\%) y en grasas totales (38\%) con dos niveles de grasas saturadas (15 vs. $8 \%$ ) respecto de una dieta base de $50 \%$ carbohidratos, $13 \%$ proteína y $38 \%$ grasa total con $15 \%$ de grasas saturadas. Los autores encontraron que la dieta alta en grasas saturadas resultó en mayores concentraciones de LDL-C patrón $A$ ( $>=25 \mathrm{~nm}$ ) que es menos riesgoso, pero no de patrón $B(<=25 \mathrm{~nm})$ más pequeño y denso, asociado a mayores riesgos de EVC.

Finalmente, Dinicolantonio (36) cuestiona severamente las recomendaciones nutricionales que promueven dietas bajas en grasas, y particularmente aquellas que reemplazan las grasas saturadas por carbohidratos o bien por AGPI, argumentando que el reemplazo de los AGS por AGPI ( $\omega-6)$ aumentaría el riesgo de ECV. Agrega que los riesgos potenciales de reemplazar las grasas saturadas por carbohidratos son: un aumento de LDL-C patrón B; cambio hacia un perfil general lipídico aterogénico (menor HDL-C, aumento de triglicéridos y aumento de la relación $\mathrm{ApoB} / \mathrm{ApoA} 1)$; pequeñas mejoras en la tolerancia a la glucosa, grasa corporal, peso, inflamación y marcadores trombogénicos; y un aumento en la incidencia de obesidad y diabetes. Por otra parte, señala que los potenciales daños de reemplazar las grasas saturadas por AGPI ( $\omega-6)$ incluyen: aumento en el riesgo de cáncer; aumento de oxidación de LDL-C; una reducción de HDL-C; aumento en el riesgo de ECV, eventos cardiovasculares, muerte debido a enfermedades cardíacas y muertes en general.

\section{CONCLUSIÓN}

La teoría de los lípidos y las recomendaciones nutricionales sugeridas en base a esta no han logrado los resultados esperados de reducir las muertes por enfermedades cardiovasculares. Asimismo, no existe evidencia científica que permita asegurar la existencia de una relación causal entre consumo de grasas saturadas presentes en productos cárnicos y lácteos con estas enfermedades. Por el contrario, estos productos contribuyen con ácidos grasos beneficiosos para la salud de las personas, en especial aquellos provenientes de sistemas producción pastoriles, como es en Chile.

\section{RESUMEN}

Las recomendaciones nutricionales de los últimos 40 años han promovido la reducción del consumo grasas, calorías y particularmente ácidos grasos saturados (AGS), enfatizando un menor consumo de carnes rojas y productos lácteos altos en materia grasa debido a su asociación con enfermedades cardiovasculares (ECV), siendo reemplazados por azúcares y carbohidratos refinados. Existe consenso en que la concentración de AGS en el plasma sanguíneo, particularmente ácido palmítico, se asocia con un mayor riesgo de ECV y ataques al corazón. Sin embargo, no hay evidencias de causalidad entre ECV y consumo de AGS, planteando dudas razonables sobre la existencia de una relación entre el consumo de AGS con los presentes en el plasma sanguíneo, y con el riesgo de ECV. La presente revisión plantea evidencia científica que demuestra que la incorporación de grasas en la dieta, específicamente grasas de origen animal, aporta importantes beneficios para la salud de las personas, en especial cuando se acompaña de una reducción en el consumo de carbohidratos. Asimismo, se plantea que los productos de rumiantes y sus grasas contribuyen con ácidos grasos beneficiosos para la salud de las personas, en especial aquellos provenientes de sistemas de producción pastoriles como los ácidos vaccénico, ruménico y linolénico.

Palabras clave: omega-3, colesterol, ácido linoleico conjugado. 


\section{BIBLIOGRAFÍA}

1. Keys A. Coronary heart disease in seven countries. Circulation, 1970. 41(1):186-195.

2. Harcombe Z, Baker J S, Cooper S M, Davies B, Sculthorpe N, DiNicolantonio $J \mathrm{~J}$, and Grace F. Evidence from randomised controlled trials did not support the introduction of dietary fat guidelines in 1977 and 1983: a systematic review and meta-analysis. Open Heart, 2015. 2(1):1-7.

3. Nordøy A. Dietary fatty acids and coronary heart disease. Lipids, 1999. 34(1):19-22.

4. McGuire M A and McGuire M K. Conjugated linoleic acid (CLA): A ruminant fatty acid with beneficial effects on human health. J Animal Sci, 2000. 77(E-Suppl):1-8.

5. Ascherio $A$ and Willett $W$ C. Health effects of trans fatty acids T Ame J of Clin Nutr. 1997; 66(4):1006-10.

6. Miettinen T A, Naukkarinen $V$, Huttunen J $K$, Mattila $S$, and Kumlin T. Fatty-acid composition of serum lipids predicts myocardial infarction. 1982; 285:993-6.

7. Willett, W.C. Dietary fats and coronary heart disease. J Intern Med, 2012. 272(1):13-24.

8. FAO. Fats and fatty acids in human nutrition. Report of an expert consultation. Food and Nutrition Paper 91, Rome. 2010: p. 180.

9. Ng S W, Slining M M, and Popkin B M. Use of caloric and noncaloric sweeteners in US consumer packaged foods, 2005-2009. J Acad Nutri Dietet, 2012. 112(11):1828-34.

10. WHO. Cardiovascular diseases (CVDs). Fact Sheet 317. Reviewed June 2016 2016; Available from: http://wWw. who.int/mediacentre/factsheets/fs317/en/.

11. Ng M, Fleming T, Robinson M, et al. Global, regional, and national prevalence of overweight and obesity in children and adults during 1980-2013: a systematic analysis for the Global Burden of Disease Study 2013. The Lancet, 2014. 384(9945): 766-81.

12. Juul F and Hemmingsson E. Trends in consumption of ultraprocessed foods and obesity in Sweden between 1960 and 2010. Public Health Nutr. 2015. FirstView: 1-12.

13. Ministerio de Salud de Chile. Chilean National Health Survey 2009-2010. 2010. Available from: http://web.minsal.cl/portal/url/item/bcb03d7bc28b64dfe040010165012d23.pdf.

14. Azar A, Franetovic $G$, Martínez $M$, and Santos H. Individual, social and environmental determinants of overweight and obesity in adolescent Chile. Rev Med Chil. 2015; 143:598-605.

15. Hoenselaar R. Saturated fat and cardiovascular disease: the discrepancy between the scientific literature and dietary advice. Nutrition, 2012. 28(2):118-23.

16. Lamarche $B$ and Couture $P$. It is time to revisit current dietary recommendations for saturated fat. Appl Physiol Nutr Metab, 2014. 39(12):1409-11.

17. González C. Chile ranks fourth in international rankings calorie intake. La Tercera, COPESA: Santiago, Chile. 2015, p. 46-47.

18. ODEPA. Evolution of apparent consumption of major food in Chile. 2015. Available from: http://www.odepa. cl/wp-content/files_mf/1411487196evolucionConsumoA parenteAlimentos.pdf.

19. WHO. Sugars intake for adults and children. 2015, World Health Organization: Geneva. p. 1-59.

20. ODEPA. Milk Bulletin year 2014. 2015, Santiago, Chile.

21. Esnaola V. Yogurt market situation in Chile. 2012, ODEPA: Santiago, Chile.

22. ODEPA. Milk Buletin year 2010, 2011, p. 1-10. Santiago, Chile.
23. Departamento de Estadísticas e Información de Salud. Some causes mortality by region from 2000 to 2011, MINSAL, Available from: http://www.deis.cl/wp-content/ uploads/2014/04/0-Chile.x/sx.

24. Saxena, U. and I.J. Goldberg. Endothelial cells and atherosclerosis: lipoprotein metabolism, matrix interactions, and monocyte recruitment. Current Opinion Lipidol, 1994. 5(5):316-22.

25. Enig M G. Know your Fats: The complete primer for understanding the nutrition of fats, pils, and cholesterol. 2000, Silver Spring, MD: Bethesda Press. 358.

26. Tarlow M J. Cholesterol and diet. Arch Dis Childh, 1989; 64(5):647-8.

27. Chan J K and McDonald B E. Reducing Cholesterol Intake: Are the recommendations valid? Canadian Family Phys, 1991; 37:673-7.

28. United State Department of Agriculture (USDA) and D.O.H.A.H.S. (HHS). Scientific Report of the 2015 Dietary Guidelines Advisory Committee. Advisory report to the Secretary of Health and Human Services and the Secretary of Agriculture. 2015. p. 571.

29. Krauss $R$ M. Dietary and genetic effects on low-density lipoprotein heterogeneity. Ann Rev Nutr, 2001. 21(1):28395.

30. Expert Panel on Detection Evaluation and Treatment of High Blood Cholesterol in Adults. Executive summary of the third report of the national cholesterol education program (NCEP) expert panel on detection, evaluation, and treatment of high blood cholesterol in adults (Adult Treatment Panel III). JAMA, 2001; 285(19):2486-97.

31. Krauss $R$ M. Dense low density lipoproteins and coronary artery disease. Ame J Cardiol, 1995. 75(6):53B-57B.

32. Jakobsen $M U, O^{\prime}$ Reilly $E J$, Heitmann $B L$, Pereira $M A$, $B a ̈ l t e r ~ K$, et al. Major types of dietary fat and risk of coronary heart disease: a pooled analysis of 11 cohort studies. Ame J Clin Nutr, 2009. 89(5):1425-32.

33. Raatz $S K$, Bibus D M, Thomas $W$, and Kris-Etherton $P M$. Total fat intake modifies plasma fatty acid composition in humans. J Nutr, 2001. 131(2):231-4.

34. King I $B$, Lemaitre $R N$, and Kestin $M$. Effect of a low-fat diet on fatty acid composition in red cells, plasma phospholipids, and cholesterol esters: investigation of a biomarker of total fat intake. Ame J Clin Nutr, 2006. 83(2):227-36.

35. Forsythe $C E$, Phinney $S D$, Fernandez $M L$, Quann $E E_{\text {, }}$ Wood R, Bibus D M, Kraemer WJ, Feinman R D, and Volek J S. Comparison of low fat and low carbohydrate diets on circulating fatty acid composition and markers of inflammation. Lipids, 2008. 43(1):65-77.

36. DiNicolantonio $\mathrm{J} J$. The cardiometabolic consequences of replacing saturated fats with carbohydrates or Omega- 6 polyunsaturated fats: Do the dietary guidelines have it wrong? Open Heart, 2014. 1(1):1-3.

37. Hu F B and Willett W C. Optimal diets for prevention of coronary heart disease. JAMA, 2002. 288(20):2569-78.

38. Rice $B H$, Kraft J, Destaillats $F$, Bauman $D E$, and Lock $A$ $L$. Ruminant-produced trans-fatty acids raise plasma $H D L$ particle concentrations in intact and ovariectomized female Hartley Guinea pigs. J Nutr, 2012. 142(9):1679-83.

39. Lacroix É, Charest A, Cyr A, Baril-Gravel L, LebeufY, Paquin $P$, Chouinard $P Y$, Couture $P$, and Lamarche B. Randomized controlled study of the effect of a butter naturally enriched in trans fatty acids on blood lipids in healthy women. Ame J Clin Nutr, 2012. 95(2):318-25.

40. Lock A L, Corl B A, Barbano D M, Bauman D E, and Ip C. 
The anticarcinogenic effect of trans-11 18:1 is dependent on its conversion to cis-9, trans-11 CLA by delta 9-desaturase in rats. J. Nutr. 2004. 134(10):2698-704.

41. Chardigny J-M, Destaillats $F$, Malpuech-Brugère $C$, Moulin J, Bauman D E, et al. Do trans fatty acids from industrially produced sources and from natural sources have the same effect on cardiovascular disease risk factors in healthy subjects? Results of the trans Fatty Acids Collaboration (TRANSFACT) study. Am Clin Nutr. 2008. 87(3):558-66.

42. Jensen $R G$. The composition of bovine milk lipids: January 1995 to December 2000. J Dairy Sci, 2002; 85(2):295-350.

43. Laben, R.C. Factors Responsible for Variation in Milk Composition. J Dairy Sci, 1963. 46(11):1293-301.

44. Sanhueza J C, Nieto S K, and Valenzuela A B. Conjugated linoleic acid: a fatty acid with isomerism trans potentially beneficial. Rev Chil Nutr, 2002. 29:98-105.

45. Parodi P W. Milk fat in human nutrition. Australian J Dairy Technol, 2004. 59(1):3-59.

46. Chilliard, Y, Ferlay A, Mansbridge $R$ M, and Doreau $M$. Ruminant milk fat plasticity: nutritional control of saturated, polyunsaturated, trans and conjugated fatty acids. Ann. Zootech. 2000. 49:181-205.

47. Vargas-Bello-Pérez $E$ and Garnsworthy $P$ C. Trans fatty acids and their role in the milk of dairy cows. Ciencia Invest Agraria, 2013. 40(3):449-73.

48. Vargas-Bello-Pérez $E$, Fehrmann-Cartes $K$, Iniguez-Gonzalez $G$, Toro-Mujica P, and Garnsworthy P C. Short communication: Chemical composition, fatty acid composition, and sensory characteristics of Chanco cheese from dairy cows supplemented with soybean and hydrogenated vegetable oils. J Dairy Sci, 2015. 98(1):111-7.

49. Vargas-Bello-Pérez E, Vera R R, Aguilar C, Lira R, Pena I, and Fernandez J. Feeding olive cake to ewes improves fatty acid profile of milk and cheese. Animal Feed Sci Technol. 2013. 184:94-9.

50. Livingstone $K M$, Lovegrove J A, and Givens D I. The impact of substituting SFA in dairy products with MUFA or PUFA on CVD risk: evidence from human intervention studies. Nutr Res Rev. 2012. 25:193-206.

51. Shingfield $K J$ and Griinari J M. Role of biohydrogenation intermediates in milk fat depression. European Journal of Lipid Sc Technol. 2007. 109:799-816.

52. Katan $M B$, Zock $P L$, and Mensink $R P$. Trans fatty acids and their effects on lipoproteins in humans. Ann Rev Nutr, 1995. 15:473-93.

53. Pan American Health Organization. Healthy oils and the elimination of industrially produced trans fatty acids in the Americas: initiative for the prevention and control of chronic diseases. 2008. Available from: http://www. healthycaribbean.org/nutrition_and_diet/documents/ TransFats.pdf.

54. Morales R, Lanuza F, Subiabre I, Carvajal A M, Canto F, and Ungerfeld E M. A comparison of milk fatty acid profile among three different dairy production systems in Los Ríos disctrict, Chile. Arch Med Veterinaria, 2015. 47:281-92.

55. Mapiye $C$, Vahmani $P, M l a m b o ~ V$, Muchenje V, Dzama $K$, Hoffman $L C$, and Dugan M E R. The trans-octadecenoic fatty acid profile of beef: Implications for global food and nutrition security. Food Res Internat, 2015.

56. Sofi F, Buccioni A, Cesari F, Gori A M, Minieri S, et al. Effects of a dairy product (pecorino cheese) naturally rich in cis-9, trans-11 conjugated linoleic acid on lipid, inflammatory and haemorheological variables: A dietary intervention study. Nutrition, Metab Cardiovas Dis, 2010. 20(2):117-124.

57. Jaudszus $A$, Jahreis $G$, Schlörmann $W$, Fischer J, Kramer $R$, et al. Vaccenic acid-mediated reduction in cytokine production is independent of $c 9, t 11-C L A$ in human peripheral blood mononuclear cells. Biochim Biophys Acta (BBA) - Molecular Cell Biol Lipids, 2012. 1821(10):1316-22.

58. Field $C J$, Blewett $H$ H, Proctor $S$, and Vine D. Human health benefits of vaccenic acid. Applied Physiol, Nutr Metab, 2009. 34(5):979-91.

59. Wang $Y$, Jacome-Sosa $M M$, and Proctor $S D$. The role of ruminant trans fat as a potential nutraceutical in the prevention of cardiovascular disease. Food Res Internat, 2012. 46(2):460-8.

60. Dilzer $A$ and Park Y. Implication of conjugated linoleic acid (CLA) in human health. Critical Rev Food Sci Nutr. 2011; 52(6):488-513.

61. Morales $R$, Folch C, Iraira S, Teuber N, and Realini C E. Nutritional quality of beef produced in Chile from different production systems. Chilean J Agricul Res. 2012; 72(1):80-86.

62. Larraín $R$ and $E$ Vargas-Bell-Pérez. Composition of cuts of meat national beef. 2013, Pontificia Universidad Católica de Chile and FIA: Santiago, Chile. p. 35.

63. Roussell M A, Hill A M, Gaugler $T L$, West $S G$, Heuvel J $P$, Alaupovic $P$, Gillies $P J$, and Kris-Etherton $P$ M. Beef in an optimal lean diet study: effects on lipids, lipoproteins, and apolipoproteins. Am J Clin Nutr, 2012. 95(1):9-16.

64. Haring B, Gronroos N, Nettleton J A, Wyler von Ballmoos M C, Selvin E, and Alonso A. Dietary protein intake and coronary heart disease in a large community based cohort: Results from the atherosclerosis risk in communities (ARIC) Study. PLoS ONE, 2014. 9(10): p. e109552.

65. Scott $L W$, Dunn J, Pownall $H J$, and et al. Effects of beef and chicken consumption on plasma lipid levels in hypercholesterolemic men. Arch Intern Med, 1994; 154(11):1261-67.

66. Gilmore $L A$, Walzem $R L$, Crouse $S F$, Smith $D R$, Adams $T H$, Vaidyanathan $V$, Cao $X$, and Smith $S$ B. Consumption of high-oleic acid ground beef increases HDL-cholesterol concentration but both high- and low-oleic acid ground beef decrease HDL particle diameter in normocholesterolemic men. J Nutr, 2011. 141(6):1188-94.

67. Parks EJ and Hellerstein M K. Carbohydrate-induced hypertriacylglycerolemia: historical perspective and review of biological mechanisms. Ame J Clin Nutr, 2000. 71(2):412-33.

68. Volek $J S$ and Feinman $R D$. Carbohydrate restriction improves the features of Metabolic Syndrome. Metabolic Syndrome may be defined by the response to carbohydrate restriction. Nutr Metab (Lond), 2005. 2:31.

69. Volek J S, Phinney S D, Forsythe C E, Quann E E, Wood $R J$, et al. Carbohydrate Restriction has a More Favorable Impact on the Metabolic Syndrome than a Low Fat Diet. Lipids, 2009. 44(4):297-309.

70. Faghihnia $N$, Mangravite $L M$, Chiu S, Bergeron $N$, and Krauss $R M$. Effects of dietary saturated fat on $L D L$ subclasses and apolipoprotein CIII in men. Eur J Clin Nutr, 2012. 66(11):1229-33.

71. Roche H M, Noone E, and Gibney A N. Conjugated linoleic acid: a novel therapeutic nutrient? Nutr Res Rev. 2001; 14:173-87. 